\title{
Dynamical and chemical properties of the "starless" core L1014
}

\author{
A. Crapsi ${ }^{1,2}$, C. H. DeVries ${ }^{1}$, T. L. Huard ${ }^{1}$, J.-E. Lee ${ }^{3}$, P. C. Myers ${ }^{1}$, N. A. Ridge ${ }^{1}$, T. L. Bourke ${ }^{1}$, N. J. Evans II ${ }^{3}$ \\ J. K. Jørgensen ${ }^{1,4}$, J. Kauffmann ${ }^{5}$, C. W. Lee ${ }^{6}$, Y. L. Shirley ${ }^{7}$, and C. H. Young ${ }^{3}$ \\ 1 Harvard-Smithsonian Center for Astrophysics, 60 Garden Street, Cambridge, MA 02138, USA \\ 2 Università degli Studi di Firenze, Dipartimento di Astronomia e Scienza dello Spazio, Largo E. Fermi 5, 50125 Firenze, Italy \\ e-mail: crapsi@arcetri.astro.it \\ 3 University of Texas at Austin, 1 University Station C1400, Austin, TX 78712-0259, USA \\ ${ }^{4}$ Leiden Observatory, PO Box 9513, 2300 RA Leiden, The Netherlands \\ 5 Max-Planck-Institut für Radioastronomie (MPIfR), Bonn, Germany \\ 6 Korea Astronomy and Space Science Institute, 61-1 Hwaam-dong, Yusung-gu, Daejon 305-348, Korea \\ 7 National Radio Astronomical Observatory, PO Box 0, Socorro, NM 87801, USA
}

Received 22 November 2004 / Accepted 28 January 2005

\begin{abstract}
Spitzer Space Telescope observations of a point-like source, L1014-IRS, close to the dust peak of the low-mass dense core L1014, have raised questions about its starless nature. These show the presence of an object with colors expected for an embedded protostar with the implication that L1014-IRS would be the lowest luminosity isolated protostar known, and an ideal target with which to test star formation theories at the low mass end.

In order to study its molecular content and to search for the presence of a molecular outflow, we mapped L1014 in at least one transition of ${ }^{12} \mathrm{CO}, \mathrm{N}_{2} \mathrm{H}^{+}, \mathrm{HCO}^{+}$, CS, and of their isotopologues ${ }^{13} \mathrm{CO}, \mathrm{C}^{18} \mathrm{O}, \mathrm{C}^{17} \mathrm{O}, \mathrm{N}_{2} \mathrm{D}^{+}$, and $\mathrm{H}^{13} \mathrm{CO}^{+}$, using the Five College Radio Astronomy Observatory (FCRAO), the IRAM $30 \mathrm{~m}$ antenna, and the Caltech Submillimeter Observatory (CSO). The data show physical and chemical properties in L1014 typical of moderately evolved dense cores: i.e. $\mathrm{H}_{2}$ central density of a few $10^{5}$ molecules $\mathrm{cm}^{-3}$, estimated mass of $\sim 2 M_{\odot}$, CO integrated depletion factor less than $10, N\left(\mathrm{~N}_{2} \mathrm{H}^{+}\right) \simeq 6 \times 10^{12} \mathrm{~cm}^{-2}$, $N\left(\mathrm{~N}_{2} \mathrm{D}^{+}\right) / N\left(\mathrm{~N}_{2} \mathrm{H}^{+}\right)$equal to $10 \%$, and relatively broad $\mathrm{N}_{2} \mathrm{H}^{+}$and $\mathrm{N}_{2} \mathrm{D}^{+}$lines $\left(0.35 \mathrm{~km} \mathrm{~s}^{-1}\right)$. Infall signatures and significant velocity shifts between optically thick and optically thin tracers are not observed in the line profiles.

No classical signatures of molecular outflow are found in the ${ }^{12} \mathrm{CO}$ and ${ }^{13} \mathrm{CO}$ FCRAO observations. In particular, no high velocity wings are found, and no well-defined blue-red lobes of ${ }^{12} \mathrm{CO}$ emission are seen in the channel maps. Sensitive, higher resolution observations will clarify the presence of a molecular outflow on a smaller scale than that probed by our observations.
\end{abstract}

Key words. ISM: clouds - ISM: evolution - ISM: individual objects: L1014 - ISM: molecules - ISM: jets and outflows stars: formation

\section{Introduction}

Starless cores are cold $(\sim 10-20 \mathrm{~K})$ and dense $\left(>10^{4} \mathrm{~cm}^{-3}\right)$ condensations of gas and dust in which no sign of a central protostellar object has been found. Previously, the easiest way to determine if dust emission was associated with a protostellar object was to search for a source emitting at mid-infrared wavelengths in the IRAS catalogue (see Beichman et al. 1986). This technique was obviously limited by the IRAS sensitivity and angular resolution.

A good example of this limitation is represented by IRAM 04191. Although André et al. (1999) found a clear sign of a class 0 object represented by a collimated bipolar outflow departing from the core peak, IRAS does not show a point source towards the dust emission peak. Moreover, the CS line they observed towards the nucleus confirmed star formation activity showing a clear double peaked profile with the blue peak brighter than the red one. This spectral asymmetry has been recognized as an indicator of systematic inward motion (Snell \& Loren 1977; Zhou 1992; Tafalla et al. 1998; Lee et al. 1999).

In the same fashion, we present here the case of L1014. This core, listed as an opacity class 6 object in the Lynds (1962) catalogue, lies -0.25 below the galactic plane, has a line of sight velocity of $\sim 4 \mathrm{~km} \mathrm{~s}^{-1}$ with respect to the local standard of rest, and is projected just $10^{\prime}$ south of another dark globule B362 ${ }^{1}$. These cores can be seen in the Digital Sky Survey optical image presented in Fig. 1, where we have overlaid visual extinction contours derived from near-infrared color excesses of background stars listed in the 2MASS catalog using the NICE technique (e.g. Lada et al. 1994; Alves et al. 1998).

\footnotetext{
1 We note that B362 and L1014 were called "L1014-1" and "L1014-2" in Lee \& Myers (1999). In the present paper we keep the original names as in Barnard et al. (1927) and Lynds (1962).
} 


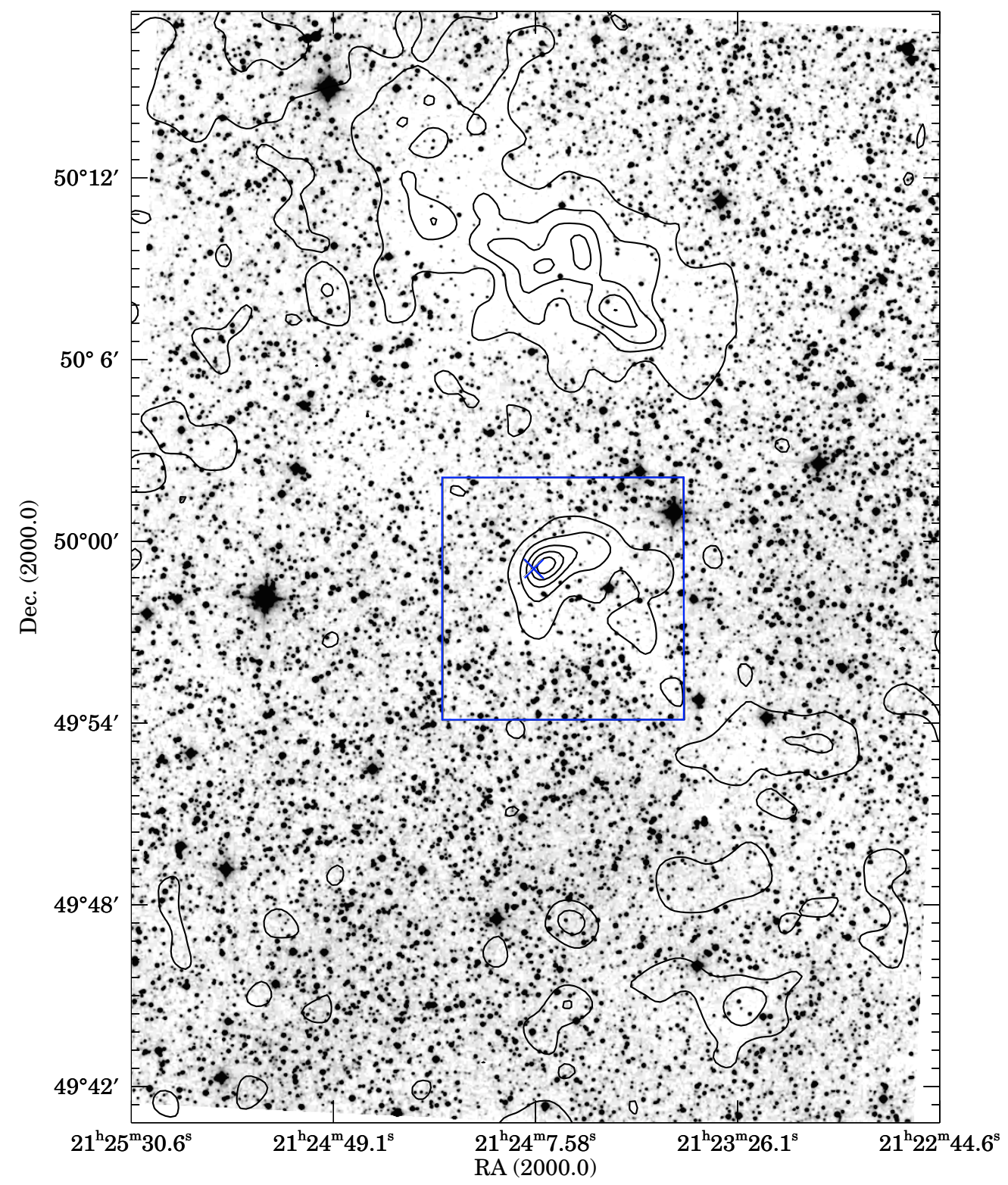

Fig. 1. Optical image of the $27^{\prime} \times 37^{\prime}$ field around L1014 from the Digital Sky Survey. Overlaid onto the image are contours of beam-averaged visual extinction, following the NICE technique (e.g., Lada et al. 1994; Alves et al. 1998) of convolving line-of-sight measurements of the $H-K$ color excesses of 2MASS sources with a $30^{\prime \prime}$ Gaussian beam. The contours are drawn at $A_{\mathrm{V}}=[3,5,7,9,11]$ magnitudes. Our FCRAO observations mapped much of this region in $\mathrm{CO}(1-0)$ and ${ }^{13} \mathrm{CO}(1-0)$, while the $\mathrm{N}_{2} \mathrm{H}^{+}(1-0)$, $\mathrm{CS}(2-1)$ and $\mathrm{C}^{18} \mathrm{O}(1-0)$ observations covered the area represented by the rectangle in the figure. L1014 is seen in the center of the field, while B362 can be seen 10' north of L1014. The cross indicates the position of L1014-IRS, the point source observed with the Spitzer Space Telescope consistent with an embedded protostar.

L1014 was included in a survey for infall asymmetry in starless cores performed while observing $\operatorname{CS}(2-1)$ and $\mathrm{N}_{2} \mathrm{H}^{+}(1-0)$ (Lee et al. 1999); given the weak detection for $\mathrm{CS}$, no $\mathrm{N}_{2} \mathrm{H}^{+}$observations were attempted. The dust continuum from L1014 was first detected at $260 \mu \mathrm{m}$ using the NASA Kuiper Airborne Observatory (KAO, Keene 1981). Subsequently an $850 \mu \mathrm{m}$ emission map (Visser et al. 2001) taken with SCUBA showed a dust emission peak at $\left(21^{\mathrm{h}} 24^{\mathrm{m}} 07^{\mathrm{s}} .6,49^{\circ} 59^{\prime} 02^{\prime \prime}, \mathrm{J} 2000\right)$ coinciding with the visual extinction peak. These authors then performed an unsuccessful search for high velocity gas, observing ${ }^{12} \mathrm{CO}(2-1)$ at five points around the dust peak with a sensitivity of $0.3 \mathrm{~K} \mathrm{~km} \mathrm{~s}^{-1}$ in a $0.2 \mathrm{~km} \mathrm{~s}^{-1}$ channel.
The Spitzer Space Telescope (hereafter Spitzer) observed L1014 in December 2003 as part of the Legacy program "From Molecular Cores to Planet Forming Disks" (Evans et al. 2003). Surprisingly, a strong $(81.8 \pm 16 \mathrm{mJy})$ point source was detected with MIPS at $24 \mu \mathrm{m}$ towards the center of L1014 (Young et al. 2004), coinciding with both the $850 \mu \mathrm{m}$ peak (Visser et al. 2001) and $1.2 \mathrm{~mm}$ peak (Young et al. 2004; Kauffmann et al., in preparation). This object, referred to as L1014-IRS, is also clearly detected in each of the 4 IRAC bands (3.6, 4.5, 5.8 , and $8.0 \mu \mathrm{m})$ and at $70 \mu \mathrm{m}$ with MIPS; and, most importantly, it shows a Spectral Energy Distribution (SED) compatible with an embedded protostar that has an effective temperature of $700 \pm 300 \mathrm{~K}$ according to the IRAC data at $\lambda \leq 8 \mu \mathrm{m}$. 
Table 1. FCRAO settings and parameters.

\begin{tabular}{lcccccc}
\hline \hline $\begin{array}{l}\text { Line } \\
(1)\end{array}$ & $\begin{array}{c}\text { Frequency } \\
(2)\end{array}$ & $\begin{array}{c}H P B W \\
(3)\end{array}$ & $\begin{array}{c}T_{\text {SYs }} \\
(4)\end{array}$ & $\begin{array}{c}\Delta v_{\text {res }} \\
(5)\end{array}$ & $\begin{array}{c}\text { Vel. cov. } \\
(6)\end{array}$ & $\begin{array}{c}\text { Mapped region } \\
(7)\end{array}$ \\
\hline $\mathrm{N}_{2} \mathrm{H}^{+}(1-0)$ & 93.1737725 & 54 & 280 & 0.157 & $-94 \rightarrow 57$ & $5^{\prime} \times 5^{\prime}$ \\
$\mathrm{CS}(2-1)$ & 97.980953 & 52 & 280 & 0.149 & $-90 \rightarrow 55$ & $5^{\prime} \times 5^{\prime}$ \\
$\mathrm{C}^{18} \mathrm{O}(1-0)$ & 109.782173 & 46 & 280 & 0.133 & $-60 \rightarrow 70$ & $5^{\prime} \times 5^{\prime}$ \\
${ }^{13} \mathrm{CO}(1-0)$ & 110.201370 & 46 & 440 & 0.133 & $-81 \rightarrow 45$ & $20^{\prime} \times 32^{\prime}$ \\
${ }^{12} \mathrm{CO}(1-0)$ & 115.271203 & 44 & 920 & 0.127 & $-78 \rightarrow 43$ & $20^{\prime} \times 32^{\prime}$ \\
\hline
\end{tabular}

Note: Column (2) line rest frequency (GHz); Col. (3) Half Power Beam Width ("); Col. (4) System Temperature (K, main beam scale); Col. (5) Channel Spacing $\left(\mathrm{km} \mathrm{s}^{-1}\right)$; Col. (6) Usable velocity coverage $\left(\mathrm{km} \mathrm{s}^{-1}\right)$; Col. (7) RA and Dec extension of the mapped region.

Considering that L1014-IRS is the only source visible at $70 \mu \mathrm{m}$ in the $5^{\prime} \times 5^{\prime}$ field observed with the Spitzer and that L1014 is the only starless core present in the same region, Young et al. (2004) concluded that a chance alignment of the two sources was unlikely although not impossible. Naively, dividing the Spitzer field of view into squared cells with a side of $30^{\prime \prime}$, the chance probability of having both L1014 and L1014-IRS in the same cell would be $1 \%$. Although we will consider the chance alignment as an alternative explanation, we will devote more discussion to the hypothesis of association since the weight of the evidence supports it. Assuming a distance of $200 \mathrm{pc}$ to the core, Young et al. (2004) determine a luminosity of $0.09 L_{\odot}$ for L1014-IRS. From the bolometric temperature $\left(T_{\text {bol }}=50 \mathrm{~K}\right)$ and the ratio between bolometric and submillimeter luminosity $\left(L_{\mathrm{bol}} / L_{\mathrm{smm}}=20\right)$, Young et al. (2004) classified L1014-IRS as a class 0 protostar (see André 1994, for a review of class 0 properties).

Outflows are a ubiquitous tracer of protostellar activity (André 1994; Richer et al. 2000); and so, in an attempt to support the classification of L1014-IRS as a class 0 protostar, we deepened the search for high velocity ${ }^{12} \mathrm{CO}$ with a better combination of resolution and sensitivity than in past searches. We report here observations of ${ }^{12} \mathrm{CO},{ }^{13} \mathrm{CO}, \mathrm{C}^{18} \mathrm{O}$, $\mathrm{C}^{17} \mathrm{O}, \mathrm{CS}, \mathrm{N}_{2} \mathrm{H}^{+}, \mathrm{N}_{2} \mathrm{D}^{+}, \mathrm{HCO}^{+}$, and $\mathrm{H}^{13} \mathrm{CO}^{+}$aimed at surveying the molecular content of L1014 and at studying its kinematical properties.

\section{Observations}

Observations were performed during March and April 2004 using the 32-pixel SEQUOIA focal plane array mounted on the Five College Radio Astronomy Observatory (FCRAO) $14 \mathrm{~m}$ telescope. We mapped $\mathrm{L} 1014$ in $\mathrm{CO}(1-0),{ }^{13} \mathrm{CO}(1-0)$, $\mathrm{C}^{18} \mathrm{O}(1-0), \mathrm{N}_{2} \mathrm{H}^{+}(1-0)$, and $\mathrm{CS}(2-1)$ using the on-the-fly position switching mode. Adopted frequencies, telescope half power beam widths, system temperatures, channel spacings, and the size of the mapped area are given in Table 1. Temperatures were converted to the main beam brightness scale according to the efficiencies tabulated in the FCRAO web page $^{2}$. Data were reduced and convolved onto a $20^{\prime \prime}$ spaced grid (circa Nyquist sampling) using the otftool software

\footnotetext{
2 http://www-astro.phast . umass. edu/ fcrao/observer/ status $14 \mathrm{~m} \cdot \mathrm{html}$ \#ANTENNA
}

(Heyer et al. 2001) ${ }^{3}$ available at FCRAO and then analysed using CLASS, the spectral line analysis software of IRAM and Observatoire de Grenoble (see Buisson et al. 2002, for a manual). Angular offsets throughout this paper are relative to the $1.2-\mathrm{mm}$ continuum peak position $\left(21^{\mathrm{h}} 24^{\mathrm{m}} 07^{\mathrm{s}} .5,49^{\circ} 59^{\prime} 05^{\prime \prime}\right.$, J2000), which is $4^{\prime \prime}$ south of L1014-IRS position.

Complementary higher-resolution observations of $\mathrm{N}_{2} \mathrm{H}^{+}(1-0),(3-2), \mathrm{N}_{2} \mathrm{D}^{+}(1-0),(2-1),(3-2)$, and $\mathrm{C}^{17} \mathrm{O}(1-0)$ were obtained in August 2004 using the IRAM-30 m telescope. The observations consisted of a 5-pointings cross spaced by $20^{\prime \prime}$ around the $1.2-\mathrm{mm}$ peak position for $\mathrm{N}_{2} \mathrm{H}^{+}(1-0), \mathrm{N}_{2} \mathrm{D}^{+}(1-0)$, and $\mathrm{N}_{2} \mathrm{D}^{+}(3-2)$, while $\mathrm{N}_{2} \mathrm{H}^{+}(3-2)$ and $\mathrm{N}_{2} \mathrm{D}^{+}(2-1)$ were observed only at the $(0,0),(20,0)$, and $(-20,0)$, and $\mathrm{C}^{17} \mathrm{O}(1-0)$ observed only at the peak position. These data were taken in frequency switching mode using the facility autocorrelator as the backend. Information about the telescope parameters (channel spacing, $H P B W$, system temperatures) are reported in Table 2. Intensities were converted in the main beam brightness scale using the efficiencies reported on the IRAM web site ${ }^{4}$.

Finally, we used the Caltech Submillimeter Observatory in August 2004 to obtain $\mathrm{HCO}^{+}(3-2), \mathrm{H}^{13} \mathrm{CO}^{+}(3-2)$, and $\mathrm{HCN}(3-2)$ spectra towards the central position, and a small map in $\mathrm{C}^{18} \mathrm{O}(2-1)$ and $\mathrm{C}^{17} \mathrm{O}(2-1)$. Spectra were obtained in position switching mode. Although the single pointing observations were performed 6 " south of L1014-IRS, the $\sim 30^{\prime \prime}$ beam allows us to consider them as relative to the infrared source position. The conversion factor to the main beam temperature $\left(\eta_{230 \mathrm{GHz}}=0.64\right)$ was evaluated from observations of planets. Other informations for the telescope set-up are reported in Table 2.

The pointing accuracy for all our observations was measured as $\approx 4-6^{\prime \prime}$.

\section{Results}

\subsection{Maps}

In Fig. 2, we present integrated intensity emission maps of $\mathrm{L} 1014\left(V_{\mathrm{LSR}} \simeq 4.2 \mathrm{~km} \mathrm{~s}^{-1}\right)$ in $\mathrm{N}_{2} \mathrm{H}^{+}(1-0), \mathrm{CS}(2-1)$, and

\footnotetext{
${ }^{3}$ http://www-astro.phast. umass.edu/ fcrao/library/ manuals/otfmanual.html

${ }^{4}$ http://www.iram.fr/IRAMES/telescope/ telescopeSummary/telescope_summary.html
} 
Table 2. IRAM-30 $\mathrm{m}$ and CSO telescope settings and parameters.

\begin{tabular}{lcccccc}
\hline \hline $\begin{array}{l}\text { Line } \\
(1)\end{array}$ & $\begin{array}{c}\text { Frequency } \\
(2)\end{array}$ & $\begin{array}{c}H P B W \\
(3)\end{array}$ & $\begin{array}{c}T_{\text {SYS }} \\
(4)\end{array}$ & $\begin{array}{c}\Delta v_{\text {res }} \\
(5)\end{array}$ & $\begin{array}{c}\text { Vel. cov. } \\
(6)\end{array}$ & $\begin{array}{c}N_{\text {obs }} \\
(7)\end{array}$ \\
\hline \multicolumn{7}{c}{ IRAM-30 m observations } \\
\hline $\mathrm{N}_{2} \mathrm{H}^{+}(1-0)$ & 93.1737725 & 26 & 165 & 0.021 & $-10 \rightarrow 16$ & 5 \\
$\mathrm{~N}_{2} \mathrm{H}^{+}(3-2)$ & 279.511863 & 9 & 1500 & 0.042 & $-16 \rightarrow 12$ & 3 \\
$\mathrm{~N}_{2} \mathrm{D}^{+}(1-0)$ & 77.109626 & 32 & 155 & 0.025 & $-14 \rightarrow 20$ & 5 \\
$\mathrm{~N}_{2} \mathrm{D}^{+}(2-1)$ & 154.217137 & 16 & 300 & 0.026 & $-10 \rightarrow 11$ & 3 \\
$\mathrm{~N}_{2} \mathrm{D}^{+}(3-2)$ & 231.321966 & 11 & 440 & 0.050 & $-16 \rightarrow 16$ & 5 \\
$\mathrm{C}^{17} \mathrm{O}(1-0)$ & 112.358990 & 22 & 240 & 0.034 & $-10 \rightarrow 16$ & 1 \\
\hline & \multicolumn{7}{c}{ CSO observations } & & & \\
\hline $\mathrm{C}^{18} \mathrm{O}(2-1)$ & 219.560352 & 34 & 390 & 0.130 & $-29 \rightarrow 37$ & 13 \\
$\mathrm{C}^{17} \mathrm{O}(2-1)$ & 224.714214 & 33 & 330 & 0.128 & $-29 \rightarrow 37$ & 5 \\
$\mathrm{HCO}^{+}(3-2)$ & 267.557620 & 28 & 320 & 0.107 & $-21 \rightarrow 28$ & 1 \\
$\mathrm{H}^{13} \mathrm{CO}(3-2)$ & 260.255478 & 29 & 300 & 0.110 & $-24 \rightarrow 32$ & 1 \\
$\mathrm{HCN}(3-2)$ & 265.886434 & 28 & 500 & 0.054 & $-24 \rightarrow 31$ & 1 \\
\hline
\end{tabular}

Note: Column (2) line rest frequency (GHz); Col. (3) Half Power Beam Width ("); Col. (4) System temperature (K, main beam scale); Col. (5) Channel spacing ( $\left.\mathrm{km} \mathrm{s}^{-1}\right)$; Col. (6) Usable velocity coverage $\left(\mathrm{km} \mathrm{s}^{-1}\right)$; Col. (7) Number of observed positions.



Fig. 2. 1.2-mm continuum, $\mathrm{N}_{2} \mathrm{H}^{+}(1-0), \mathrm{CS}(2-1)$ and $\mathrm{C}^{18} \mathrm{O}(1-0)$ emission towards L1014. Molecular line maps were taken at FCRAO with the exception of the $\mathrm{N}_{2} \mathrm{H}^{+}(1-0)$ map in the inset (from IRAM-30 m) and the $\mathrm{C}^{18} \mathrm{O}(2-1)$ map in the inset (from CSO) The 1.2-mm continuum was taken from Young et al. (2004) and it was observed with IRAM-30 m. First contour and contour spacings are $0.08 \mathrm{~K} \mathrm{~km} \mathrm{~s}^{-1}$ for the FCRAO molecular data and $4 \mathrm{mJy} /\left(11^{\prime \prime} \text { beam }\right)^{-1}$ for the dust continuum. The white contour represents the half peak intensity in all the maps. Beam sizes are displayed at the top-left of each map in all but the 1.2-mm map. The white cross places the position of a point-like source detected by the Spitzer Space Telescope, and colors are compatible with an embedded protostar. Angular offsets are relative to the 1.2-mm continuum peak $\left(21^{\mathrm{h}} 24^{\mathrm{m}} 07.5,49^{\circ} 59^{\prime} 05^{\prime \prime}, \mathrm{J} 2000\right)$. The inset on the $\mathrm{N}_{2} \mathrm{H}^{+}(1-0)$ FCRAO map shows the 5-points observations of $\mathrm{N}_{2} \mathrm{H}^{+}(1-0)$ at IRAM-30 m. Levels start at $0.8 \mathrm{~K} \mathrm{~km} \mathrm{~s}^{-1}$ and increase by $0.3 \mathrm{~K} \mathrm{~km} \mathrm{~s}^{-1}$. The IRAM- $30 \mathrm{~m}$ beam size is shown inside that of the FCRAO. Similarly, the inset in the $\mathrm{C}^{18} \mathrm{O}(1-0)$ FCRAO map shows the 13-points map of $\mathrm{C}^{18} \mathrm{O}(2-1)$ taken at CSO. Levels start at $0.35 \mathrm{~K} \mathrm{~km} \mathrm{~s}^{-1}$ and increase by $0.1 \mathrm{~K} \mathrm{~km} \mathrm{~s}^{-1}$. The CSO beam size is shown inside that of the FCRAO. 
$\mathrm{C}^{18} \mathrm{O}(1-0)$ taken with FCRAO, the 1.2-mm continuum from Young et al. (2004), the 5-points map of $\mathrm{N}_{2} \mathrm{H}^{+}(1-0)$ taken with the IRAM-30 m, and the 13-points map of $\mathrm{C}^{18} \mathrm{O}(2-1)$ from CSO. The half maximum contour of each map is rendered in white. A white cross shows the position of L1014-IRS, the candidate protostellar object embedded in L1014 according to Young et al. (2004). The area observed with the FCRAO is $10^{\prime} \times 10^{\prime}$ in size, but only the inner $8^{\prime} \times 8^{\prime}$ region where emission was detected is shown in Fig. 2.

$\mathrm{N}_{2} \mathrm{H}^{+}(1-0)$ emission, which comes from the high density nucleus of the core, is very concentrated (FWHM $\sim 70^{\prime \prime}$, in the FCRAO observations) and peaks $\sim 10^{\prime \prime}$ west of the dust emission within the uncertainties due to the 54" FCRAO beam width and the Nyquist sampling of the observations. Since the emitting region is comparable in size to the telescope beam, some dilution is very likely. In fact, IRAM-30 m observations of $\mathrm{N}_{2} \mathrm{H}^{+}(1-0)$ with a $26^{\prime \prime}$ beam showed spectra 3 times brighter than those observed by FCRAO towards the dust continuum peak, although the emission still does not seem to be fully resolved. In these higher resolution observations, the integrated intensity peak falls $5^{\prime \prime}$ south of L1014-IRS. We evaluated the equivalent radius of the $\mathrm{N}_{2} \mathrm{H}^{+}(1-0)$ emission from FCRAO both from the square root of the area within the 50\% contour divided by $\pi$ and from the geometric mean of the semimajor and semi-minor axis, both estimates furnish $0.034 \mathrm{pc}$. This equivalent radius is among the 5 smallest radii found in Lee et al. (2001) and in Caselli et al. (2002), who observed 52 starless cores all together using FCRAO and then evaluated the equivalent radius with one of the two techniques. The integrated intensity of $\mathrm{N}_{2} \mathrm{H}^{+}(1-0)$ at peak observed with FCRAO is also lower (a factor of 2) than any other core reported in Lee et al. (2001) and Caselli et al. (2002), although the beam dilution should be kept in mind. Following Caselli et al. (2002), we calculated the virial mass of L1014 from $M_{\mathrm{vir}}\left(M_{\odot}\right)=210 r(\mathrm{pc}) \Delta v_{\mathrm{m}}^{2}\left(\mathrm{~km}^{2} \mathrm{~s}^{-2}\right)$, where $r$ is the typical radius of the $\mathrm{N}_{2} \mathrm{H}^{+}$emission from FCRAO and $\Delta v_{\mathrm{m}}^{2}$ is the velocity dispersion of the mean mass molecule (see Caselli et al. 2002, for the definition). In this way we obtained a virial mass of $2.1 M_{\odot}$, that is again among the smallest in the Caselli et al. (2002) sample. This estimate is in agreement with the mass evaluated from the extinction map in Fig. 1. In fact, using a conversion factor of $1 \times 10^{21}$ molecules $\mathrm{cm}^{-2}$ per mag of visual extinction (Bohlin et al. 1978), we obtained a mass of $1.5 M_{\odot}$ above the $A_{\mathrm{V}}=5 \mathrm{mag}$ contour.

The CS(2-1) emission is much more extended than the 1.2-mm continuum and $\mathrm{N}_{2} \mathrm{H}^{+}(1-0)$, as expected due to its lower critical density and possible depletion effects (Tafalla et al. 2002). The integrated intensity peak lies $\sim 30^{\prime \prime}$ west of the dust emission, although the same caveats about the limited resolution and sampling apply here as in the $\mathrm{N}_{2} \mathrm{H}^{+}$map.

$\mathrm{C}^{18} \mathrm{O}(1-0)$ observations reveal more structure than previous observations, showing a secondary peak at $\left(-200^{\prime \prime},-200^{\prime \prime}\right)$ from the reference position. Note that the $1.2-\mathrm{mm}$ map did not cover that area, and the $\mathrm{C}^{18} \mathrm{O}(1-0)$ integrated intensity peaks west of the continuum, although the peak is less constrained than the other tracers. The flatness of the $\mathrm{C}^{18} \mathrm{O}$ map and the offset of its peak with respect to the dust are typical features of starless cores (Tafalla et al. 2002), where CO (and its isotopologues) freeze-out onto dust grains in the high density nucleus. To test if this is the situation in L1014, we follow Caselli et al. (2002b) by evaluating the degree of CO depletion as the ratio of the canonical CO abundance $\left([\mathrm{CO}] /\left[\mathrm{H}_{2}\right] \equiv\right.$ $9.5 \times 10^{-5}$, Frerking et al. 1982) and the observed abundance derived from the ratio of $\mathrm{C}^{18} \mathrm{O}$ and $\mathrm{H}_{2}$ column densities (for details see Caselli et al. 2002b). Following Crapsi et al. (2005), the CO column density was derived under the constant excitation temperature approximation (CTEX, see Eq. (A4) in Caselli et al. 2002b) assuming that $\mathrm{C}^{18} \mathrm{O}$ emission is optically thin. In this way we derived a $\mathrm{C}^{18} \mathrm{O}$ column density of $7.8 \times 10^{14} \mathrm{~cm}^{-2}$ at the dust peak position, which increases to $8.2 \times 10^{14} \mathrm{~cm}^{-2}$ at the $\mathrm{C}^{18} \mathrm{O}$ peak position $\left(-20^{\prime \prime}, 20^{\prime \prime}\right)$. The $\mathrm{CO}$ column density can then be inferred from the local interstellar medium relative abundance value $\left([\mathrm{CO}] /\left[\mathrm{C}^{18} \mathrm{O}\right]=560\right.$; Wilson \& Rood 1994). $\mathrm{H}_{2}$ column density was inferred from the $1.2-\mathrm{mm}$ emission assuming constant dust temperature $(10 \mathrm{~K})$ and emissivity $\left(\kappa_{1.2 \mathrm{~mm}}=0.005 \mathrm{~cm}^{2} \mathrm{~g}^{-1}\right)$ similarly to Ward-Thompson et al. (1999), thereby obtaining $N\left(\mathrm{H}_{2}\right)=4 \times 10^{22} \mathrm{~cm}^{-2}$ at the dust peak position and $N\left(\mathrm{H}_{2}\right)=2.2 \times 10^{22} \mathrm{~cm}^{-2}$ at the $\mathrm{C}^{18} \mathrm{O}$ peak position. We thus found a depletion factor around 9 at the dust peak, whereas it drops to 5 at the $\mathrm{C}^{18} \mathrm{O}$ peak. These values are typical of starless cores with moderate chemical evolution (e.g. L1495, L1498, L492) with densities of about a few $10^{5} \mathrm{~cm}^{-3}$ (Crapsi et al. 2005). The $30^{\prime \prime}$ map of $\mathrm{C}^{18} \mathrm{O}(2-1)$ obtained at CSO is shown in an inset of Fig. 2. Note that the error on the integrated intensity for the points outside the peak is 2.5 times the value reported in Table 3. Similarly to $\mathrm{N}_{2} \mathrm{H}^{+}$ high-resolution observations, the $\mathrm{C}^{18} \mathrm{O}(2-1)$ from CSO seems to peak south of L1014-IRS.

While the 1.2-mm emission peak in L1014 (23 mJy $\left.\left(11^{\prime \prime} \text { beam }\right)^{-1}\right)$ is comparable in intensity to the majority of $\mathrm{N}_{2} \mathrm{H}^{+}$emitting starless cores (see e.g. Crapsi et al. 2005), it is relatively weak if compared with high-density, evolved pre-stellar cores such as L1544 (>60 mJy $\left(11^{\prime \prime} \text { beam }\right)^{-1}$, Ward-Thompson et al. 1999). Consequently, the $\mathrm{H}_{2}$ central volume density inferred from it is 6 times lower $\left(2.5 \times 10^{5} \mathrm{~cm}^{-3}\right.$ in L1014 vs. $1.4 \times 10^{6} \mathrm{~cm}^{-3}$ in L1544) when evaluated with the same technique and physical parameters as in Tafalla et al. (2002). This technique derives the density profile of the spherically symmetric core that best fits the observed $1.2-\mathrm{mm}$ continuum. Our calculations adopted a density profile model of the form $n\left(\mathrm{H}_{2}\right)=n_{0} /\left(1+r / r_{0}\right)^{\alpha}$ to find, in addition to the central density, the radius of the "flat" region $\sim 20^{\prime \prime}$ and the asymptotic power law index $\sim 2.7$. Note that the central $\mathrm{H}_{2}$ density value $\left(1.5 \times 10^{5} \mathrm{~cm}^{-3}\right)$ given in Young et al. (2004) was evaluated using a dust opacity equal to $0.0104 \mathrm{~cm}^{2} \mathrm{~g}^{-1}$.

\subsection{Spectra towards dust peak}

The spectra observed towards the dust emission peak with FCRAO are shown in Fig. 3 while those observed with IRAM- $30 \mathrm{~m}$ are shown in Fig. 4 and those taken at CSO in Fig. 5. Gaussian fits were performed using the CLASS fitting procedure, and results are presented in Table 3 . In the case of lines with hyperfine structure, all the hyperfine components were simultaneously fit, which also furnishes the opacity of 
Table 3. Line parameters at the 1.2-mm continuum peak position from line profile fitting.

\begin{tabular}{|c|c|c|c|c|}
\hline $\begin{array}{l}\text { Line } \\
\text { (1) }\end{array}$ & $\begin{array}{c}\text { Intensity } \\
\text { (2) }\end{array}$ & $\begin{array}{c}V_{\mathrm{LSR}} \\
(3)\end{array}$ & $\begin{array}{l}\Delta V \\
(4)\end{array}$ & $\begin{array}{c}\tau \\
(5)\end{array}$ \\
\hline \multicolumn{5}{|c|}{ FCRAO spectra } \\
\hline $\mathrm{N}_{2} \mathrm{H}^{+}(1-0)$ & $0.52 \pm 0.03$ & $4.239 \pm 0.014$ & $0.354 \pm 0.033$ & $4.6 \pm 3.2$ \\
\hline $\mathrm{CS}(2-1)$ & $0.41 \pm 0.02$ & $4.279 \pm 0.014$ & $0.677 \pm 0.031$ & $\ldots$ \\
\hline $\mathrm{C}^{18} \mathrm{O}(1-0)$ & $0.84 \pm 0.02$ & $4.224 \pm 0.005$ & $0.484 \pm 0.012$ & $\ldots$ \\
\hline${ }^{13} \mathrm{CO}(1-0)$ & $3.18 \pm 0.07$ & $4.258 \pm 0.008$ & $0.864 \pm 0.019$ & $\ldots$ \\
\hline${ }^{12} \mathrm{CO}(1-0)$ & $9.30 \pm 0.17$ & $4.211 \pm 0.022$ & $2.261 \pm 0.049$ & $\ldots$ \\
\hline \multicolumn{5}{|c|}{ IRAM-30 m spectra } \\
\hline $\mathrm{N}_{2} \mathrm{H}^{+}(1-0)$ & $1.850 \pm 0.032$ & $4.242 \pm 0.004$ & $0.354 \pm 0.009$ & $6.1 \pm 0.8$ \\
\hline $\mathrm{N}_{2} \mathrm{H}^{+}(3-2)$ & $0.263 \pm 0.036$ & $4.270 \pm 0.035$ & $0.443 \pm 0.058$ & $<0.100$ \\
\hline $\mathrm{N}_{2} \mathrm{D}^{+}(1-0)$ & $0.203 \pm 0.010$ & $4.248 \pm 0.007$ & $0.284 \pm 0.020$ & $2.1 \pm 1.6$ \\
\hline $\mathrm{N}_{2} \mathrm{D}^{+}(2-1)$ & $0.182 \pm 0.015$ & $4.273 \pm 0.010$ & $0.307 \pm 0.029$ & $<0.100$ \\
\hline $\mathrm{N}_{2} \mathrm{D}^{+}(3-2)$ & $0.066 \pm 0.014$ & $4.303 \pm 0.044$ & $0.315 \pm 0.128$ & $<0.100$ \\
\hline $\mathrm{C}^{17} \mathrm{O}(1-0)$ & $0.476 \pm 0.022$ & $4.229 \pm 0.009$ & $0.310 \pm 0.027$ & $2.4 \pm 1.5$ \\
\hline \multicolumn{5}{|c|}{ CSO spectra } \\
\hline $\mathrm{C}^{18} \mathrm{O}(2-1)$ & $0.641 \pm 0.036$ & $4.270 \pm 0.019$ & $0.575 \pm 0.048$ & $1.4^{a}$ \\
\hline $\mathrm{C}^{17} \mathrm{O}(2-1)$ & $0.344 \pm 0.035$ & $4.092 \pm 0.023$ & $0.489 \pm 0.051$ & $<0.100$ \\
\hline $\mathrm{HCO}^{+}(3-2)$ & $0.316 \pm 0.015$ & $4.410 \pm 0.019$ & $0.819 \pm 0.041$ & $\ldots$ \\
\hline $\mathrm{H}^{13} \mathrm{CO}^{+}(3-2)$ & $0.025 \pm 0.019$ & $\ldots$ & $\ldots$ & $\ldots$ \\
\hline $\operatorname{HCN}(3-2)^{b}$ & $<0.03$ & $\ldots$ & $\ldots$ & $\ldots$ \\
\hline
\end{tabular}

Note: Column (2) $\int T_{\mathrm{MB}} \mathrm{d} V\left(\mathrm{~K} \mathrm{~km} \mathrm{~s}^{-1}\right)$ (in presence of hyperfine structure; we integrated over all the components; ${ }^{12} \mathrm{CO}$ and ${ }^{13} \mathrm{CO}$ lines were integrated between 1 and $7 \mathrm{~km} \mathrm{~s}^{-1}$ and 3 and $5.5 \mathrm{~km} \mathrm{~s}^{-1}$ respectively); Col. (3) Rest velocity ( $\left.\mathrm{km} \mathrm{s}^{-1}\right)$; Col. (4) Full width half maximum (km s${ }^{-1}$ ); Col. (5) Sum of the opacity of all the hyperfine components.

${ }^{a}$ Opacity was evaluated from the opacity of $\mathrm{C}^{17} \mathrm{O}(2-1)$ and assumed the relative abundance in Wilson \& Rood (1994).

${ }^{b}$ Upper limit on integrated intensity was evaluated for a line-width equal to $\mathrm{H}^{13} \mathrm{CO}^{+}(3-2)$.

the line in the presence of high $\mathrm{S} / \mathrm{N}$ spectra. The $\mathrm{N}_{2} \mathrm{H}^{+}(1-0)$ line widths $\left(0.35 \mathrm{~km} \mathrm{~s}^{-1}\right)$ are slightly broader than the prototype of evolved starless core L1544 $\left(0.31 \mathrm{~km} \mathrm{~s}^{-1}\right.$, Caselli et al. 2002a), suggesting a combination of nonthermal and systematic motions in the inner nucleus of L1014. Using the CTEX approximation on the higher resolution (IRAM-30 m) data, we found a column density at the integrated intensity peak position of $N\left(\mathrm{~N}_{2} \mathrm{H}^{+}\right)=6 \pm 2 \times 10^{12} \mathrm{~cm}^{-2}$, comparable to other starless cores moderately evolved in a chemical sense like e.g. L1512, L1498, L1495, L1517B (Crapsi et al. 2005), and a factor of 2-3 less than in IRAM 04191, the other very low-luminosity class 0 object known (Belloche \& André 2004). Similarly, the $\mathrm{N}_{2} \mathrm{D}^{+}$column density derived from the $\mathrm{N}_{2} \mathrm{D}^{+}(1-0)$ and $(2-1)$ IRAM-30 m spectra yielded a value of $=6 \pm 1 \times 10^{11} \mathrm{~cm}^{-2}$ from both lines. The deuterium fractionation at the peak of L1014 was thus found to be $10 \%$, a value higher than the average starless core (Crapsi et al. 2005). $\mathrm{N}_{2} \mathrm{H}^{+}$ and $\mathrm{N}_{2} \mathrm{D}^{+}$column densities were also calculated in the Large Velocity Gradient approximation (see e.g. Crapsi et al. 2005) and yielded values of $5.3 \times 10^{12} \mathrm{~cm}^{-2}$ and $5.2 \times 10^{11} \mathrm{~cm}^{-2}$ respectively. This approach also furnishes an estimate of the $\mathrm{H}_{2}$ volume density from observations of multiple rotational transitions; in this way we obtained $n\left(\mathrm{H}_{2}\right) \simeq 1.9 \times 10^{5} \mathrm{~cm}^{-3}$ from $\mathrm{N}_{2} \mathrm{H}^{+}$and $n\left(\mathrm{H}_{2}\right) \simeq 4.0 \times 10^{5} \mathrm{~cm}^{-3}$ from $\mathrm{N}_{2} \mathrm{D}^{+}$. Both estimates are consistent within their errors with the central density evaluated from the dust continuum.

The ${ }^{12} \mathrm{CO}(1-0)$ and ${ }^{13} \mathrm{CO}(1-0)$ profiles are much more asymmetric than the other lines, and their observed line widths $\left(\sim 2 \mathrm{~km} \mathrm{~s}^{-1}\right.$ and $\left.\sim 1 \mathrm{~km} \mathrm{~s}^{-1}\right)$ are broader $\left(\sim 0.5 \mathrm{~km} \mathrm{~s}^{-1}\right)$. This occurrence is not consistent with line broadening caused by opacity effects alone, as already noted by Robert \& Pagani (1993). A possible explanation could be found by considering that $\mathrm{CO}$ and ${ }^{13} \mathrm{CO}$ emission comes from a combination of emissions by randomly-moving, low-density parts of the cloud with different velocities along the line of sight and with larger turbulence. $\mathrm{C}^{17} \mathrm{O}(1-0)$ opacity was found to be $2.4 \pm 1.5$ from hyperfine structure fitting, although the low signal-to-noise ratio lets us think that this might just be an upper limit. In fact, the total column density derived from other indicators is totally inconsistent with such high opacity for $\mathrm{C}^{17} \mathrm{O}$.

Interestingly, our CS(2-1) spectra do not show the asymmetric double-peaked structure, with the blue peak stronger than the red one, as is typical of the cores undergoing infall (Zhou 1992; Mardones et al. 1997; Lee et al. 1999). 

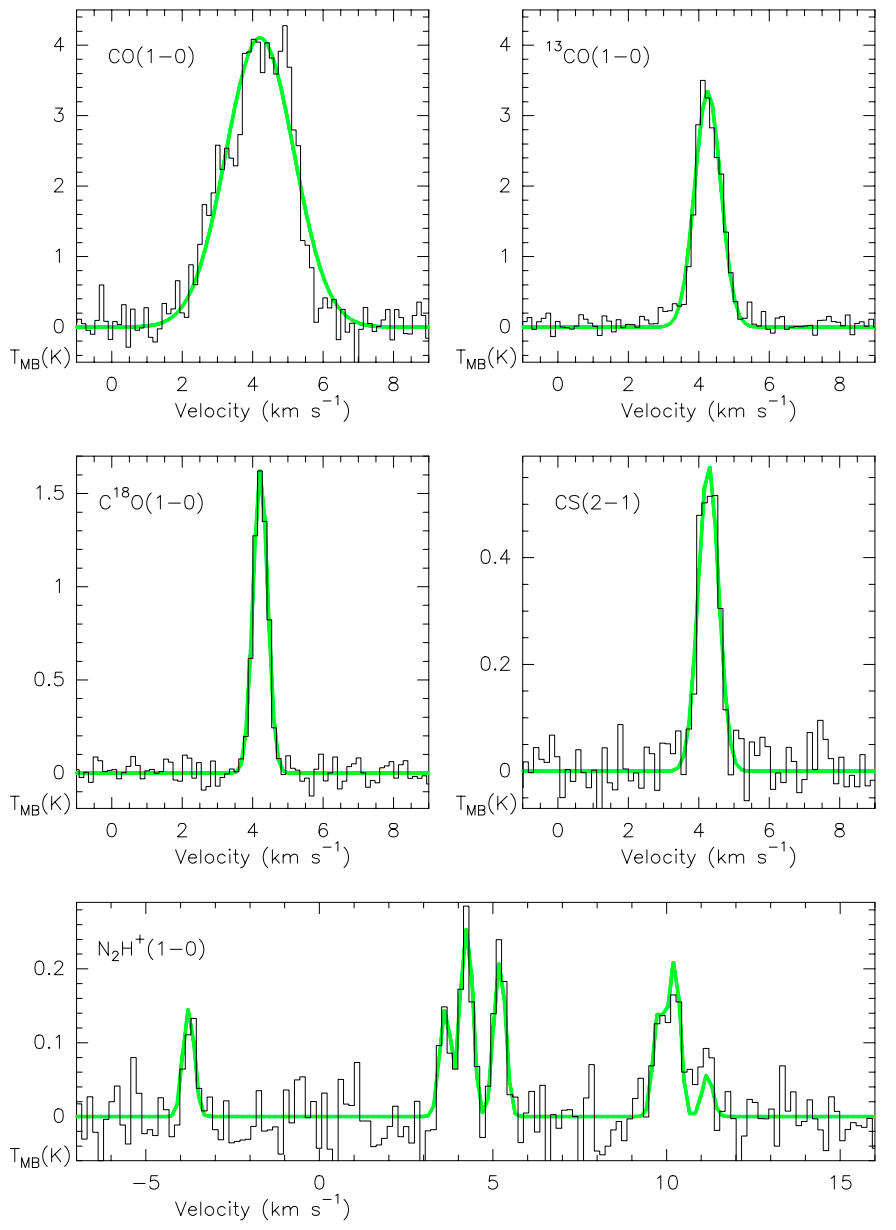

Fig. 3. $\quad \mathrm{CO}(1-0), \quad{ }^{13} \mathrm{CO}(1-0), \quad \mathrm{C}^{18} \mathrm{O}(1-0), \quad \mathrm{CS}(2-1), \quad$ and $\mathrm{N}_{2} \mathrm{H}^{+}(1-0)$ spectra observed with FCRAO towards the $1.2-\mathrm{mm}$ peak of L1014. Gaussian (or hyperfine in the case of $\mathrm{N}_{2} \mathrm{H}^{+}(1-0)$ ) fits are plotted.

This feature was clearly seen in IRAM 04191 (André et al. 1999). Moreover, the difference in line velocity between $\mathrm{CS}$ and $\mathrm{N}_{2} \mathrm{H}^{+}$in units of the $\mathrm{N}_{2} \mathrm{H}^{+}(1-0)$ line width $\left(\delta V_{\mathrm{CS}}=\left(V_{\mathrm{CS}}-V_{\mathrm{N}_{2} \mathrm{H}^{+}}\right) / \Delta V_{\mathrm{N}_{2} \mathrm{H}^{+}}\right)$, which is supposed to gauge the extended infall motions of the core (Mardones et al. 1997; Lee et al. 1999), is very small in L1014 (0.11 \pm 0.04$)$.

In contrast, $\mathrm{HCO}^{+}(3-2)$ observations show the opposite asymmetry revealing a red peak brighter than the blue peak (see Fig. 5). This line profile can be produced by a rotating, expanding, or pulsating core (Lada et al. 2003). The velocity of the self absorbed feature corresponds to the velocity of the optically thin $\mathrm{C}^{18} \mathrm{O}(2-1)$ line as predicted by the models. Given the moderate signal to noise ratio of the present data, it is necessary to confirm these indications with higher sensitivity and higher resolution observations and to retrieve a map of $\mathrm{HCO}^{+}$in order to search for spatial patterns for the expansion asymmetry.

We failed to detect $\mathrm{H}^{13} \mathrm{CO}^{+}(3-2)$ and $\mathrm{HCN}(3-2)$ towards L1014-IRS with upper limits of 0.02 and $0.03 \mathrm{~K} \mathrm{~km} \mathrm{~s}^{-1}$, respectively (assuming line widths of $1.2 \mathrm{~km} \mathrm{~s}^{-1}$ at base).
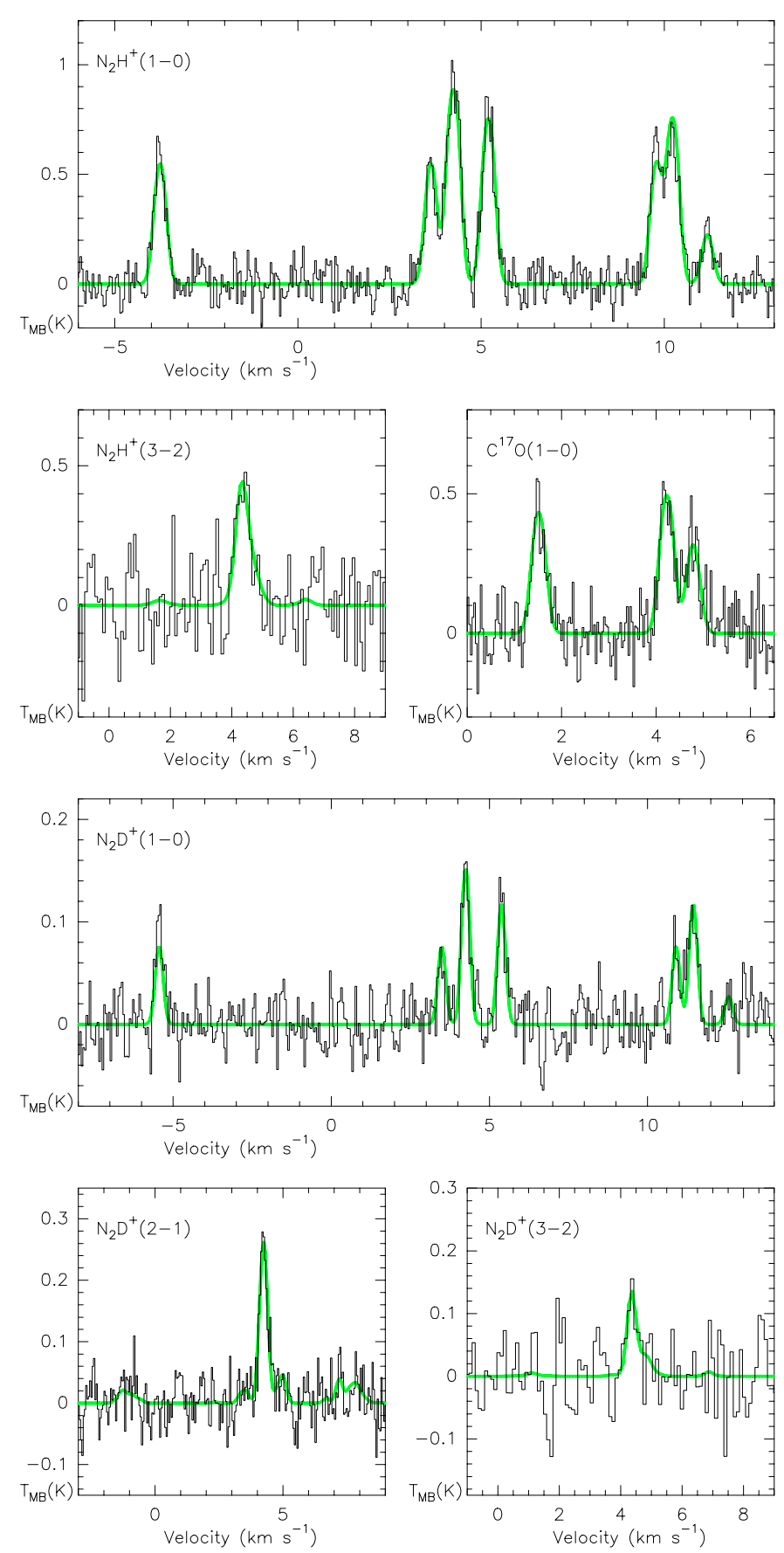

Fig. 4. $\mathrm{N}_{2} \mathrm{H}^{+}(1-0), \mathrm{N}_{2} \mathrm{H}^{+}(3-2), \mathrm{C}^{17} \mathrm{O}(1-0), \mathrm{N}_{2} \mathrm{D}^{+}(1-0), \mathrm{N}_{2} \mathrm{D}^{+}(2-1)$, and $\mathrm{N}_{2} \mathrm{D}^{+}(3-2)$ spectra observed with the IRAM-30 m towards the 1.2-mm peak of L1014. Hyperfine fits are plotted.

\subsection{The search for outflow in L1014}

In Fig. 6, we present channel maps of $\mathrm{CO}(1-0)$ (top) and ${ }^{13} \mathrm{CO}(1-0)$ (bottom) in a range of velocities, $-9 \mathrm{~km} \mathrm{~s}^{-1}$ to $15 \mathrm{~km} \mathrm{~s}^{-1}$, centered around L1014's typical velocity $\left(\sim 4.2 \mathrm{~km} \mathrm{~s}^{-1}\right)$. Emission was integrated in $2 \mathrm{~km} \mathrm{~s}^{-1}$ intervals. No CO emission was found anywhere in the mapped area between $-35 \mathrm{~km} \mathrm{~s}^{-1}$ and $-9 \mathrm{~km} \mathrm{~s}^{-1}$ or between $15 \mathrm{~km} \mathrm{~s}^{-1}$ and $25 \mathrm{~km} \mathrm{~s}^{-1}$, i.e at velocities departing more than $10 \mathrm{~km} \mathrm{~s}^{-1}$ from the L1014 rest velocity. The $1 \sigma$ errors for the ${ }^{12} \mathrm{CO}$ and 


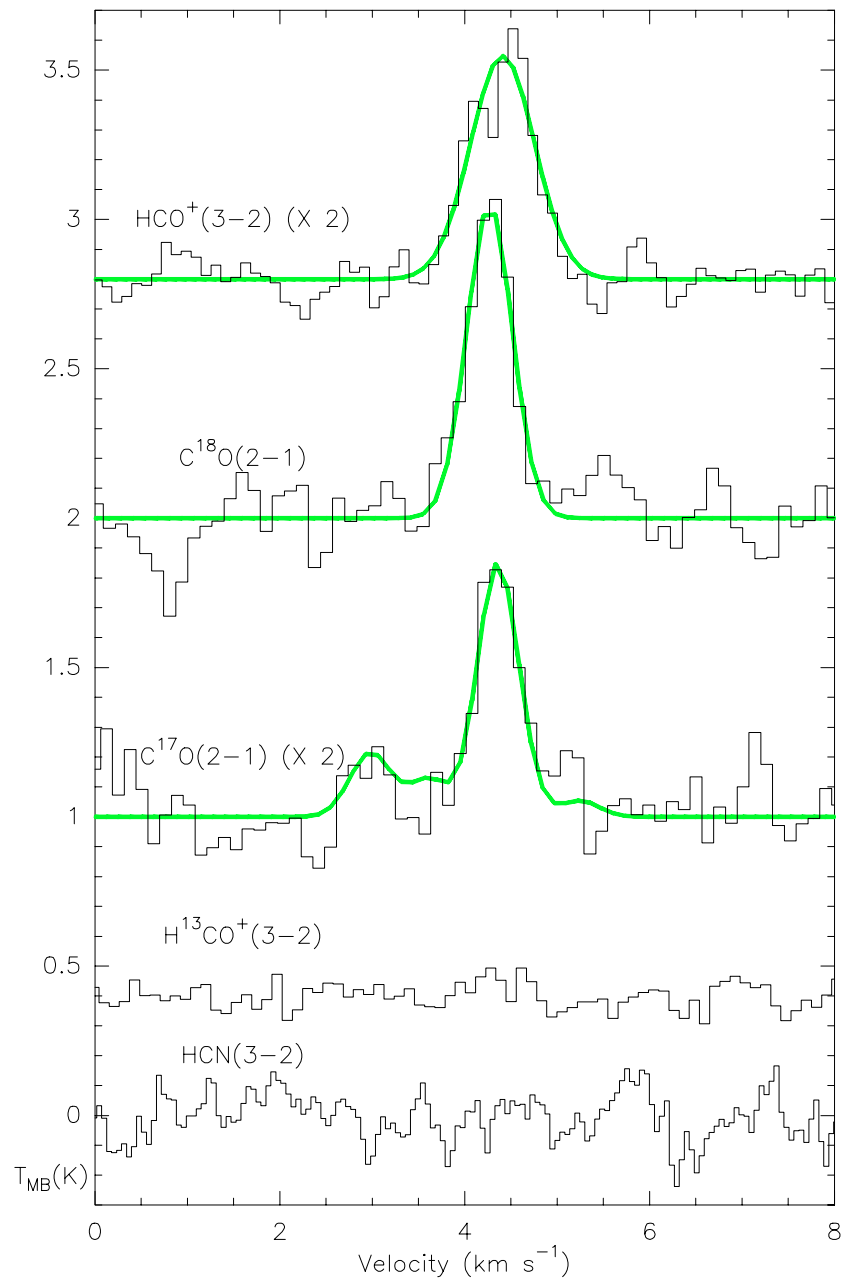

Fig. 5. $\mathrm{C}^{18} \mathrm{O}(2-1), \mathrm{C}^{17} \mathrm{O}(2-1), \mathrm{HCO}^{+}(3-2), \mathrm{H}^{13} \mathrm{CO}^{+}(3-2)$, and $\mathrm{HCN}(3-2)$ spectra observed with the CSO towards the 1.2-mm peak of L1014. Gaussian or hyperfine fits are plotted. The asymmetric shape of $\mathrm{HCO}^{+}(3-2)$ is consistent with outward motions of the gas.

${ }^{13} \mathrm{CO}$ channel maps are $0.06 \mathrm{~K} \mathrm{~km} \mathrm{~s}^{-1}$ and $0.03 \mathrm{~K} \mathrm{~km} \mathrm{~s}^{-1}$ respectively, less than the lowest contours adopted in Fig. 6.

Using IRAM 04191 (André et al. 1999) as a guide, we searched for ${ }^{12} \mathrm{CO}(1-0)$ wing emission at velocities $3 \mathrm{~km} \mathrm{~s}^{-1}<$ $\left|V-V_{0}\right|<9 \mathrm{~km} \mathrm{~s}^{-1}$. No wing-like emission was found in this range (see maps labeled $-5,-3,-1$ and 7, 9, 11 in Fig. 6).

Nevertheless, given the very low luminosity of L1014-IRS, we also examined the possibility of less energetic wings. The most likely outflow feature present in L1014 is along position angle $45^{\circ}$ (measured East of North) where we can see red emission in the SW lobe between 5 and $7 \mathrm{~km} \mathrm{~s}^{-1}$ and blue emission in the NE part in the bin 1 to $3 \mathrm{~km} \mathrm{~s}^{-1}$. This axis was identified mainly from the jet-like appearance of the "red lobe", but the fact that this feature is also seen in the ${ }^{13} \mathrm{CO}(1-0)$ and $\mathrm{C}^{18} \mathrm{O}(1-0)$ emissions, which typically are not sensitive to outflows, let us think that these components arise from different parts of the cloud with different velocities along the line of sight. The presence of gas with different velocities along the same line of sight would also explain the relatively broad ${ }^{12} \mathrm{CO}$ and ${ }^{13} \mathrm{CO}$ line widths found in the entire map (see Robert \& Pagani 1993, and Sect. 3.2). A peculiar feature of
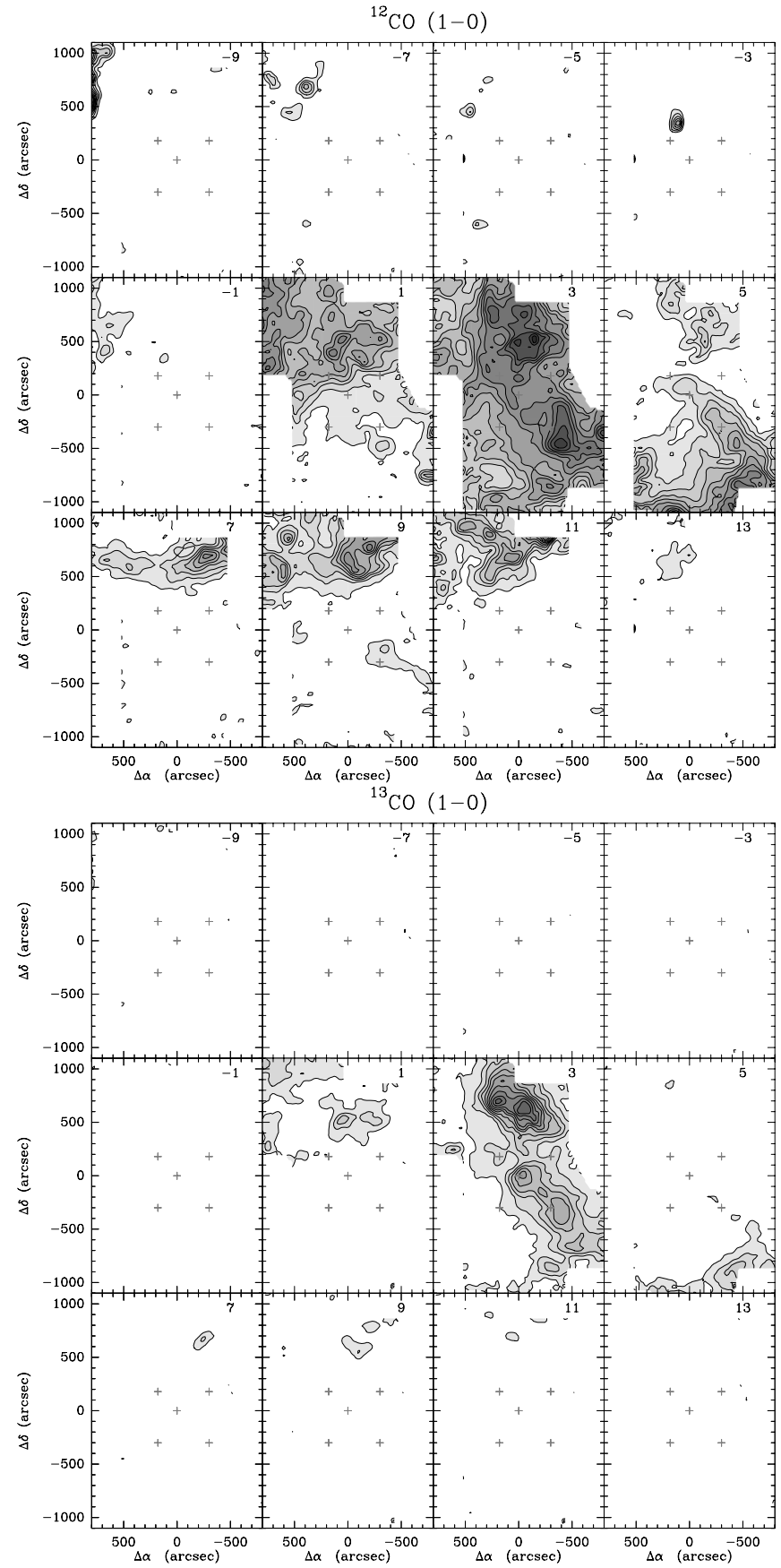

Fig. 6. Channel maps of the $\mathrm{CO}(1-0)$ (top panel) and ${ }^{13} \mathrm{CO}(1-0)$ (bottom panel) lines around the rest velocity of L1014. First contour and contour spacing are $0.8 \mathrm{~K} \mathrm{~km} \mathrm{~s}^{-1}$ for the $\mathrm{CO}$ data and $0.05 \mathrm{~K} \mathrm{~km} \mathrm{~s}^{-1}$ for the ${ }^{13} \mathrm{CO}$ ones. Velocity bins are spaced at $2 \mathrm{~km} \mathrm{~s}^{-1}$ intervals and the starting velocity of the bin is reported in the top right corner of each map. The central cross on each map indicates the dust peak, while the other four delineate the area shown in Fig. 2.

the ${ }^{12} \mathrm{CO}$ channel maps is the presence of several small spots of emission in the NW part of the map at velocities smaller than $1 \mathrm{~km} \mathrm{~s}^{-1}$ and of one spot at $9 \mathrm{~km} \mathrm{~s}^{-1}$ in the SE. Although these may be interpreted as molecular "bullets" coming from the L1014 nucleus, their asymmetric positioning, low velocity, and large distance from the nucleus $(0.05 \mathrm{pc})$ make them very different from the prototypical cases (Bachiller 1996). 
We conclude that no classical outflow signature is present at the large scales investigated with the FCRAO. Further sensitive observations must be performed with smaller beams to probe the CO emission within the inner 45" (the FCRAO beam size). We can evaluate an upper limit on the mass of a compact outflow within the inner beam determining the CO column density under the LTE approximation; using $T_{\mathrm{ex}}=10 \mathrm{~K}$ and $\left[{ }^{12} \mathrm{CO}\right] /\left[\mathrm{H}_{2}\right]=9.5 \times 10^{-5}$, we obtained: $M_{\text {outflow }}=3.9 \times$ $10^{-4} \sigma_{I_{\text {wing }}} / f \quad\left[M_{\odot}\right]$, where $\sigma_{I_{\text {wing }}}$ is the $1 \sigma$ error on the integrated intensity of the $\mathrm{CO}$ wing and $f$ is the filling factor of the outflow in the beam. Considering an outflow of $5^{\prime \prime}$ in length and $3 \mathrm{~km} \mathrm{~s}^{-1}$ of velocity, the error on the integrated intensity of the $\mathrm{CO}$ spectrum at peak $\left(0.1 \mathrm{~K} \mathrm{~km} \mathrm{~s}^{-1}\right)$ furnish an upper limit of $\sim 2 \times 10^{-3} M_{\odot}$.

\subsection{The background component}

The Spitzer observations presented by Young et al. (2004) left open the possibility of L1014-IRS being a more massive but less embedded distant protostar aligned by chance with the L1014 nucleus. Although the relative rarity of embedded protostar and starless cores do not favour such a chance occurrence, the fact that we are looking close to the galactic plane and towards the Perseus spiral arm did not allow us to disregard it on a statistical basis alone. Although preliminary results from recent deep near-infrared observations (T. Huard et al. 2005, in prep.) and high resolution $\mathrm{CO}$ observations (T. Bourke et al. 2005 , in prep.) now seem to indicate that L1014-IRS is associated with the nearby L1014 core, in this paper we take the conservative approach and assume that the association is yet to be confirmed.

We searched for signatures of infall-outflow activity towards L1014-IRS also at the Perseus arm velocity (near $-40 \mathrm{~km} \mathrm{~s}^{-1}$ ). No $\mathrm{N}_{2} \mathrm{H}^{+}(1-0), \mathrm{CS}(2-1)$, and $\mathrm{C}^{18} \mathrm{O}(1-0)$ emissions were detected at these velocities, most likely because of beam dilution given the small scales traced by those species and the large Perseus arm distance $(2.6 \mathrm{kpc}$, Brand \& Blitz 1993). Young et al. (2004) calculated that assuming a distance of $2.6 \mathrm{kpc}$ for L1014-IRS, the protostar whose emission would fit the observed SED best would have a luminosity of $16 L_{\odot}$. Class I and class 0 protostars with comparable bolometric luminosity, like L1165 (Visser et al. 2002), L1448, and L1157 (Bachiller 1996), drive outflows of $\approx 0.3 \mathrm{pc}$ in size, corresponding to $\approx 20^{\prime \prime}$ at the Perseus arm distance. Although this size is smaller than the FCRAO beam, we performed a search for high velocity wings in the $-40 \mathrm{~km} \mathrm{~s}^{-1} \mathrm{com}$ ponent. $\mathrm{CO}$ and ${ }^{13} \mathrm{CO}$ emission is seen between $-56 \mathrm{~km} \mathrm{~s}^{-1}$ and $-36 \mathrm{~km} \mathrm{~s}^{-1}$, but no clear-cut outflow features are seen in the channel maps, similarly to the component at $4 \mathrm{~km} \mathrm{~s}^{-1}$.

\section{Discussion}

The contradiction between the relatively young evolutionary status of L1014, as derived from chemical indicators and the presence of a protostellar object embedded in its nucleus, is puzzling. L1014 shows continuum and line intensities lower than well-studied evolved starless cores; in particular, the $\mathrm{H}_{2}$ volume density, and $\mathrm{N}_{2} \mathrm{H}^{+}$and $\mathrm{N}_{2} \mathrm{D}^{+}$column densities are smaller than the average starless core, from which we did not expect L1014 to be close to star formation. Moreover, the CS observation does not indicate the presence of inward motions, and the $\mathrm{HCO}^{+}$line profile shows hints of outward motions.

On the other hand, we report also relatively high deuterium fractionation, moderate $\mathrm{CO}$ depletion, broad (if compared to other low-mass starless cores) $\mathrm{N}_{2} \mathrm{H}^{+}$and $\mathrm{N}_{2} \mathrm{D}^{+}$lines, and a very compact $\mathrm{N}_{2} \mathrm{H}^{+}$equivalent radius which are typical of more evolved objects. We speculate that either i) the very low-luminosity nature of the central source $\left(0.09 L_{\odot}\right.$, Young et al. 2004) makes its chemical and dynamical evolution different from any other observed core; or ii) that a low-mass, lowluminosity "seed" is present long before the protostellar phase in L1014 and perhaps in some other "starless" core. Boss \& Yorke (1995) and Masunaga et al. (1998) modeled the early phase of protostar formation and introduced the "first hydrostatic core" or "class -I" protostar as a short-lived precursor of class 0 . During this phase, the central temperature should reach a value of $200 \mathrm{~K}$ (3 times lower than the temperature derived by Young et al. 2004) and no outflow emission is expected. According to the duration of this phase, we could expect to detect similar sources in the nuclei of some other starless cores in future Spitzer observations. This seed might also be produced by an impulsive event, such as a sudden compression early in the core evolution, before it would otherwise form a star "spontaneously". In this sense, the presence of a protostar in L1014 seems to challenge the idea that there is a single path toward forming a protostar that all cores must follow.

Considering that L1014-IRS is classified as a class 0 protostar (Young et al. 2004) that meets the requirements of $T_{\text {bol }}<$ $70 \mathrm{~K}$ and $L_{\text {bol }} / L_{\text {smm }}<200$ (Andre et al. 1993), we expected it to show also a bipolar outflow; on the other hand our observations show that it does not have a strong, extended molecular outflow. Even IRAM 04191, which has bolometric luminosity comparable to L1014-IRS $\left(\sim 0.15 L_{\odot}\right)$, has an easily detected, very extended outflow (André et al. 1999); thus, it seems unlikely that the weakness of the central source is responsible for the lack of outflow detection. In any case, one should bear in mind that differences in inclination angle, collimation factor, age, as well as in the external environment, could make detection of the outflow more difficult even in presence of two jets with comparable momenta. We can speculate that either the accretion rate of L1014-IRS is currently too low to power the outflow or that the magnetic field that threads the disk is too weak (Bachiller 1996). Alternatively, we may have caught L1014 in the earliest phases of outflow ignition. In fact, our observations do not probe small scale outflows that would be diluted in the $45^{\prime \prime}$ FCRAO beam, in particular if the outflow is very young and therefore compact. Strong molecular outflows are a hallmark of class 0 protostar (André 1994), so it is of fundamental importance to extend the search for the outflow to a smaller scale. High-resolution observations of $\mathrm{CO}(2-1)$ from SMA are currently under analysis (T. Bourke et al. 2005, in prep.); although yet to be confirmed, these data seem to show that a very compact outflow may be present in the inner $4 "$.

The present data alone cannot conclusively rule out the possibility of chance alignment, although our search for 
background dense cores through $\mathrm{N}_{2} \mathrm{H}^{+}$and CS was negative, and no signatures of molecular outflows were found in the $\mathrm{CO}$ background component. The absence of a background dense core could be explained by the chance crossing of a $\mathrm{T}$ Tauri star born elsewhere and expelled in the direction of L1014 (see PV Cephei, Goodman \& Arce 2004). This explanation, although difficult to rule out, is even less probable than chance alignment, given additional requirements of a favourable trajectory from the parent cloud to L1014 and the coincidence in the epoch of observations. Further evidence that L1014-IRS is a young stellar object embedded within L1014 comes from deep near-infrared observations and will be presented by T. Huard et al. (in preparation).

\section{Conclusions}

We observed the starless core L1014 with the FCRAO antenna in $\mathrm{N}_{2} \mathrm{H}^{+}(1-0), \quad \mathrm{CS}(2-1), \quad \mathrm{C}^{18} \mathrm{O}(1-0),{ }^{13} \mathrm{CO}(1-0)$, and ${ }^{12} \mathrm{CO}(1-0)$, combined with literature $1.2-\mathrm{mm}$ continuum data and new $\mathrm{N}_{2} \mathrm{H}^{+}(1-0), \mathrm{N}_{2} \mathrm{H}^{+}(3-2), \mathrm{N}_{2} \mathrm{D}^{+}(1-0)$, $\mathrm{N}_{2} \mathrm{D}^{+}(2-1), \mathrm{N}_{2} \mathrm{D}^{+}(3-2)$, and $\mathrm{C}^{17} \mathrm{O}(1-0)$ observations from the IRAM-30 m, along with $\mathrm{C}^{18} \mathrm{O}(2-1), \mathrm{C}^{17} \mathrm{O}(2-1), \mathrm{HCO}^{+}(3-2)$, $\mathrm{H}^{13} \mathrm{CO}^{+}(3-2)$, and $\mathrm{HCN}(3-2)$ spectra from CSO to study its chemical status and to search for the presence of an extended molecular outflow. The results of our study are summarized below.

1. The chemical and physical properties of L1014 derived from the present observations are not typical of highly evolved low-mass starless cores. In particular, we found: molecular hydrogen volume density of $n\left(\mathrm{H}_{2}\right) \simeq 2.5 \times$ $10^{5} \mathrm{~cm}^{-3}, \mathrm{~N}_{2} \mathrm{H}^{+}$column density of $N\left(\mathrm{~N}_{2} \mathrm{H}^{+}\right) \simeq 6 \times$ $10^{12} \mathrm{~cm}^{-2}, M_{\mathrm{vir}}=2.1 M_{\odot}, \mathrm{CO}$ integrated depletion factor equal to 9 , absence of CS double peaked profile with infall signature, absence of significant velocity shifts between self-absorbed optically thick (CS) and optically thin $\left(\mathrm{N}_{2} \mathrm{H}^{+}\right)$tracers, and profile asymmetry in $\mathrm{HCO}^{+}(3-2)$ consistent with outward motions. On the other hand, we found an enhanced degree of deuterium fractionation equal to $10 \%$, which is higher than the average starless core, a very compact $\mathrm{N}_{2} \mathrm{H}^{+}$equivalent radius $(\sim 0.03 \mathrm{pc})$, and broad $\mathrm{N}_{2} \mathrm{H}^{+}$and $\mathrm{N}_{2} \mathrm{D}^{+}$lines $\left(\sim 0.3 \mathrm{~km} \mathrm{~s}^{-1}\right)$ suggestive of unresolved kinematical activity in the inner nucleus.

These diverging indications could be reconciled considering that we are observing either a very young stage of star formation or an extremely low-luminosity object exhibiting characteristics that differ significantly from previously known cases.

2. No classical signatures of molecular outflow were found towards L1014 in the FCRAO data. In particular, we did not detect high velocity wings or symmetric well-defined redblue lobes in the large scale CO channel maps, although it is important to bear in mind that the angular resolution of the present observations might be too low to detect a compact, young outflow.

Acknowledgements. We gratefully thank Dr. Paola Caselli and Dr. Arnaud Belloche for watching over the observations at the IRAM-30 m, and the referee, Dr. Laurent Pagani, for clarifying several points in the manuscript. A.C. was partly supported by NASA "Origins of Solar System Grant" (NAG 5-13050). C.W.L. acknowledges supports from the Basic Research Program (KOSEF R01-2003000-10513-0) of the Korea Science and Engineering Foundation. This work has been partly supported by NASA "Origins of Solar System Grant" (NNG04GG24G). FCRAO is supported by NSF grant AST 02-28993.

\section{References}

Alves, J., Lada, C. J., Lada, E. A., Kenyon, S. J., \& Phelps, R. 1998, ApJ, 506, 292

Andre, P., Ward-Thompson, D., \& Barsony, M. 1993, ApJ, 406, 122

André, P. 1994, The Cold Universe, 179

André, P., Motte, F., \& Bacmann, A. 1999, ApJ, 513, L57

Barnard, E. E. 1927, A Photographic Atlas of Selected Regions of the Milky Way, ed. E. B. Frost, \& M. R. Calvert (Washington: Carnegie institution of Washington)

Bachiller, R. 1996, ARA\&A, 34, 111

Beichman, C. A., Myers, P. C., Emerson, J. P., et al. 1986, ApJ, 307, 337

Belloche, A., \& André, P. 2004, A\&A, 419, L35

Brand, J., \& Blitz, L. 1993, A\&A, 275, 67

Bohlin, R. C., Savage, B. D., \& Drake, J. F. 1978, ApJ, 224, 132

Boss, A. P., \& Yorke, H. W. 1995, ApJ, 439, L55

Buisson, G., Desbats, L., Duvert, G., et al. 2002, CLASS manual Caselli, P., Walmsley, C. M., Zucconi, A., et al. 2002a, ApJ, 565, 331

Caselli, P., Walmsley, C. M., Zucconi, A., et al. 2002b, ApJ, 565, 344

Caselli, P., Benson, P. J., Myers, P. C., \& Tafalla, M. 2002c, ApJ, 572, 238

Crapsi, A., Caselli, P., Walmsley, C. M., et al. 2005, ApJ, 619, 379

Evans, N. J., Allen, L. E., Blake, G. A., et al. 2003, PASP, 115, 965

Frerking, M. A., Langer, W. D., \& Wilson, R. W. 1982, ApJ, 262, 590

Goodman, A. A., \& Arce, H. G. 2004, ApJ, 608, 831

Heyer, M. H., Narayanan, G., \& Brewer, M. K. 2001, On the Fly Mapping at the FCRAO $14 \mathrm{~m}$ Telescope, FCRAO

Keene, J. 1981, ApJ, 245, 115

Lada, C. J., Lada, E. A., Clemens, D. P., \& Bally, J. 1994, ApJ, 429, 694

Lada, C. J., Bergin, E. A., Alves, J. F., \& Huard, T. L. 2003, ApJ, 586, 286

Lee, C. W., \& Myers, P. C. 1999, ApJS, 123, 233

Lee, C. W., Myers, P. C., \& Tafalla, M. 1999, ApJ, 526, 788

Lee, C. W., Myers, P. C., \& Tafalla, M. 2001, ApJS, 136, 703

Lynds, B. T. 1962, ApJS, 7, 1

Mardones, D., Myers, P. C., Tafalla, M., et al. 1997, ApJ, 489, 719

Masunaga, H., Miyama, S. M., \& Inutsuka, S. 1998, ApJ, 495, 346

Richer, J. S., Shepherd, D. S., Cabrit, S., Bachiller, R., \& Churchwell, E. 2000, Protostars and Planets IV, 867

Ridge, N. A., \& Moore, T. J. T. 2001, A\&A, 378, 495

Robert, C., \& Pagani, L. 1993, A\&A, 271, 282

Shu, F. H., Adams, F. C., \& Lizano, S. 1987, ARA\&A, 25, 23

Snell, R. L., \& Loren, R. B. 1977, ApJ, 211, 122

Tafalla, M., Mardones, D., Myers, P. C., et al. 1998, ApJ, 504, 900

Tafalla, M., Myers, P. C., Caselli, P., Walmsley, C. M., \& Comito, C. 2002, ApJ, 569, 815

Visser, A. E., Richer, J. S., \& Chandler, C. J. 2001, MNRAS, 323, 257

Visser, A. E., Richer, J. S., \& Chandler, C. J. 2002, AJ, 124, 2756

Ward-Thompson, D., Motte, F., \& Andre, P. 1999, MNRAS, 305, 143

Wilson, T. L., \& Rood, R. 1994, ARA\&A, 32, 191

Young, C. H., Jørgensen, J. K., Shirley, Y. L., et al. 2004, ApJS, 154, 396

Zhou, S. 1992, ApJ, 394, 204 